\title{
The effect of vitamin $D$ in the evolution of experimental autoimmune encephalomyelitis
}

\author{
Vitor Almeida da Silva (EM), Vanessa C.B Mariano (EM), Stephani O Alves (EM), Fabiana F. Aquino \\ (EM), Paula G. Russini, Amanda Piffer, LeonildaM.B.Santos
}

\begin{abstract}
Vitamin D3 deficiency is associated with an increased risk of multiple sclerosis (MS) and unfavorable MS disease progression. Here we demonstrated immunomodulatory effect of vitamin $\mathrm{D}$ in Experimental autoimmune encephalomyelitis a experimental model for studying MS.
\end{abstract}

Key words: multiple sclerosis, vitamin D, EAE.

\section{Introduction}

EAE is an antigen-driven autoimmune model in which immunization against myelin autoantigens elicits strong CD4 T lymphocyte responses, which initiate its pathology with central nervous system myelin destruction. Material and Methods: EAE was induced by immunization with MOG35- 55 peptide emulsified in complete Freund adjuvant. The clinical expression of the disease was graded on a clinical scale 0-5 according to the severity of the disease. Vitamin D3 (cholecalciferol D3) Sigma Aldr. Mo, USA) was diluted in polyethylene glycol and give orally $(5 \mu \mathrm{g} / \mathrm{Kg} /$ day $)$ during 2 weeks. The control group was fed with vehicle alone (15 animal/group were studied in three independent experiments. The expression of cytokine mRNA was evaluated by quantitative RTPCR.

\section{Results and Discussion}

We demonstrated that the oral administration of vitamin $D$ reduced significantly the severity of EAE. The reduction of the severity of the disease was accompanied by the reduction of proinflammatory cytokines such as IFNyand increase of antinflammatory cytokines such as IL10 and IL-27

Score Analysis Charts

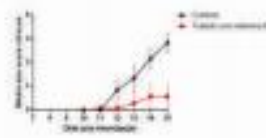

Results Real Time PCR

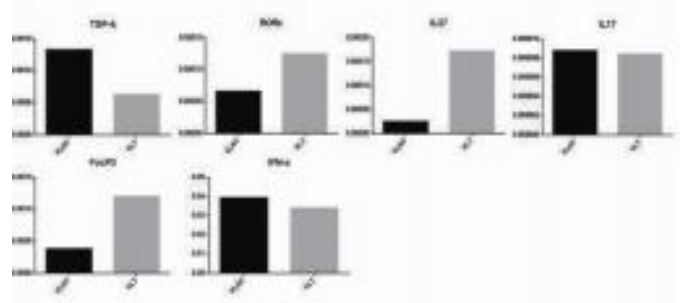

Score is the technique used to measure the animal's Disease.

\section{Conclusions}

We conclude that vitamin D3 presentimmunomodulatory effect in the $E A E$ model. The immunosuppressive mechanism may inhibit the migration of autoreactive $\mathrm{T}$ Iymphocytes into central nervous system. Moreover, thestudy suggest that supplementation with vitamin D3 may be beneficial to patients with multiple sclerosis, that present a functional vitamin $D$ receptor.

\section{Acknowledgement}

Wethankour mentor Prof. Dr. Leonilda MB dos Santos

${ }^{1}$ Reference: ${ }^{2}$ Vitamin D3 Induces IDO+ Tolerogenic DCs and Enhances Treg,Reducing the Severity of EAE 